\title{
Dynamics of Excitability over Extended Timescales in Cultured Cortical Neurons
}

\author{
Asaf Gal, ${ }^{1,4}$ Danny Eytan, ${ }^{1,5}$ Avner Wallach, ${ }^{1,2}$ Maya Sandler, ${ }^{3}$ Jackie Schiller, ${ }^{3}$ and Shimon Marom ${ }^{1,3}$ \\ ${ }^{1}$ Network Biology Research Laboratories, Lorry Lokey Interdisciplinary Center for Life Sciences and Engineering, ${ }^{2}$ Faculty of Electrical Engineering, and \\ ${ }^{3}$ Department of Physiology, Faculty of Medicine, Technion, Haifa 32000, Israel, ${ }^{4}$ The Interdisciplinary Center for Neural Computation, The Hebrew \\ University, Jerusalem 91904, Israel, and ${ }^{5}$ Rambam Medical Center, Haifa 31096, Israel
}

\begin{abstract}
Although neuronal excitability is well understood and accurately modeled over timescales of up to hundreds of milliseconds, it is currently unclear whether extrapolating from this limited duration to longer behaviorally relevant timescales is appropriate. Here we used an extracellular recording and stimulation paradigm that extends the duration of single-neuron electrophysiological experiments, exposing the dynamics of excitability in individual cultured cortical neurons over timescales hitherto inaccessible. We show that the long-term neuronal excitability dynamics is unstable and dominated by critical fluctuations, intermittency, scale-invariant rate statistics, and long memory. These intrinsic dynamics bound the firing rate over extended timescales, contrasting observed short-term neuronal response to stimulation onset. Furthermore, the activity of a neuron over extended timescales shows transitions between quasi-stable modes, each characterized by a typical response pattern. Like in the case of rate statistics, the short-term onset response pattern that often serves to functionally define a given neuron is not indicative of its long-term ongoing response. These observations question the validity of describing neuronal excitability based on temporally restricted electrophysiological data, calling for in-depth exploration of activity over wider temporal scales. Such extended experiments will probably entail a different kind of neuronal models, accounting for the unbounded range, from milliseconds up.
\end{abstract}

\section{Introduction}

The traditional paradigm in neuroscience assumes that neuronal threshold dynamics are local in time, that is, affected only by the recent history of membrane voltage, maybe one or two orders of magnitude longer than the timescale of a single action potential, not more. Accordingly, present-day mathematical models of single neurons account for spike times and spike rates over timescales that are up to hundreds of milliseconds (Hodgkin and Huxley, 1952; Izhikevich, 2003, 2004; Golomb et al., 2006; Brette et al., 2007; Badel et al., 2008; Jolivet et al., 2008; Gerstner and Naud, 2009; Kobayashi et al., 2009 and references therein). Longer temporal effects, according to the above paradigm, should be explained by either emergent collective modes or changes in connectivity at the neural population level.

However, as already pointed out almost a century ago in the pioneering work of Adrian and Zotterman (1926), temporal membrane dynamics extend far beyond hundreds of milliseconds, so much so that there is a considerable overlap between timescales of brain-behavior dynamics and timescales of excitability (Turrigiano et al., 1994; Desai et al., 1999). This is hardly

Received Sept. 16, 2010; accepted Sept. 24, 2010

This work was partially funded by grants from the Israel Science Foundation (S.M.) and the Etai Sharon Rambam Atidim Program for Excellence in Research (D.E.). We thank Erez Braun, Danni Dagan, Ron Meir, Daniel Soudry, and Noam Ziv for helpful discussions and insightful input and Eleonora Lyakhov and Vladimir Lyakhov for technical assistance in conducting the experiments.

Correspondence should be addressed to Asaf Gal, Network Biology Research Laboratories, Technion, Haifa 32000, Israel. E-mail: asaf.gal@mail.huji.ac.il.

DOI:10.1523/JNEUROSCI.4859-10.2010

Copyright $\odot 2010$ the authors $\quad$ 0270-6474/10/3016332-11\$15.00/0 surprising given the immensity of the involved cellular level processes that culminate in modulation of membrane conductances (Marom, 2010): large numbers and coupled heterogeneous collections of ion conducting proteins, which are under heavy structural and functional modulations, arising from gene expression regulation and intracellular activity-dependent signal transduction machineries. These processes, together with changes in cell morphology, practically cover any observable cell-physiology timescale.

What are the consequences of this immensity of cellular processes on single-neuron excitability in general and threshold dynamics in particular? It turns out that even when a small subset of these processes is numerically integrated in single neuron models (Lowen et al., 1999; Gilboa et al., 2005), temporally complex dynamics of spike time series are expected, including long-term correlations, broad distributions of practically every statistic of activity, and $1 / f$ spectral behaviors. Presumably, attempts to integrate more processes that modulate excitability will yield even further complexity. Although this theoretical result is congruent with measurement of long-term network-embedded singleneuron activity in vivo (Teich, 1989; Lowen and Teich, 1992, 1996; Teich et al., 1997), the interpretation of such data is limited by inherent inability to separate neuronal excitability from network effects on the observed complex dynamics. Therefore, temporally extended data from a single neuron that is isolated from network effects is wanted; such data are especially called for in light of recent interest in the functional and theoretical implications of wide temporal range dynamics at the single-neuron level (Fairhall et al., 2001; Drew and Abbott, 2006; French and 


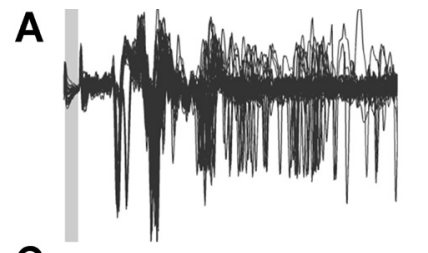

C

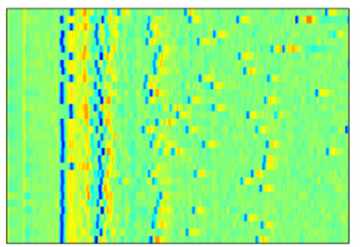

B

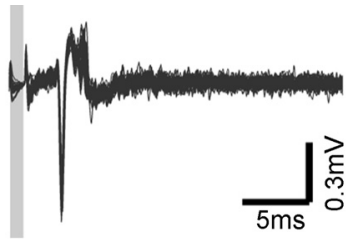

D

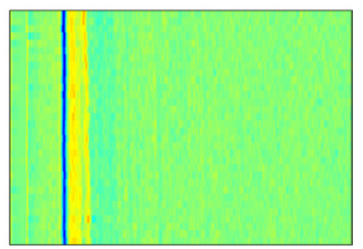

Figure 1. The effect of synaptic blockers on the activity of cultured neurons. A, Overlaid voltage traces recorded from a single electrode in response to $30 \mathrm{~min}$ of $1 \mathrm{~Hz}$ pulse stimulation, before application of synaptic blockers. The shaded area marks the stimulation period. The response contain both directly evoked spikes, as well as synaptically mediated response. $\boldsymbol{B}$, Responses from the same electrode, after the application of synaptic blockers. All reverberating and synaptically mediated responses vanished, and only the directly evoked spike is seen. $\boldsymbol{C}$, The responses in $\boldsymbol{A}$, shown as a color-coded image. Each line represents a single voltage response after a stimulation. Responses are sequentially ordered from top to bottom. $\boldsymbol{D}$, The responses of $\boldsymbol{B}$, shown as a color-coded image. The latency and shape of the directly evoked spike are more stable after the application of blockers.

Torkkeli, 2008; Lundstrom et al., 2008; Friedlander and Brenner, 2009; Marom, 2009, 2010). Here we present a series of experiments that use the robustness of extracellular stimulation and recording of neuronal responses in culture over a very long (practically unbounded) duration, thus generating millions-of-spikeslong time series. Such data lend itself to analyses of long-term statistics in temporal resolution ranging from milliseconds to days. By conducting such long experiments on synaptically isolated neurons, we show that excitability dynamics in itself is intrinsically unstable, exhibiting intermittency, critical longrange fluctuations, and broad distributions of both activity patterns and activity statistics. Keeping in mind obvious constraints on our ability to extrapolate from the cultured reduced experimental system studied here, we argue that these results question the usefulness and, indeed, the validity of scaling standard models that are used to reconstruct shortterm membrane excitability dynamics, to extended temporal and spatial regimens.

\section{Materials and Methods}

\section{Culture preparation}

Cortical neurons were obtained from newborn rats (Sprague Dawley) within $24 \mathrm{~h}$ after birth using mechanical and enzymatic procedures described in previous studies (Marom and Shahaf, 2002). The neurons were plated directly onto substrate-integrated multielectrode arrays (MEAs) and allowed to develop functionally and structurally mature networks over a time period of $2-3$ weeks. The number of neurons in a typical network is on the order of 10,000. The preparations were bathed in Minimal Essential Medium supplemented with heat-inactivated horse serum $(5 \%)$, glutamine $(0.5 \mathrm{mM})$, glucose $(20 \mathrm{mM})$, and gentamycin (10 $\mathrm{g} / \mathrm{ml}$ ) and maintained in an atmosphere of $37^{\circ} \mathrm{C}, 5 \% \mathrm{CO}_{2}$ and $95 \%$ air in an incubator as well as during the recording phases. An array of Ti/Au/ TiN extracellular electrodes, $30 \mu \mathrm{m}$ in diameter, and spaced either 500 or $200 \mu \mathrm{m}$ from each other (Multi Channel Systems) were used. The insulation layer (silicon nitride) was pretreated with polyethyleneimine. Where indicated, synaptic transmission in the network was completely blocked by adding $20 \mu \mathrm{M}$ amino-5-phosphonovaleric acid, $10 \mu \mathrm{M}$ 6-cyano-7-nitroquinoxaline-2,3-dione, and $5 \mu \mathrm{M}$ bicucullinemethiodide to the bathing solution.

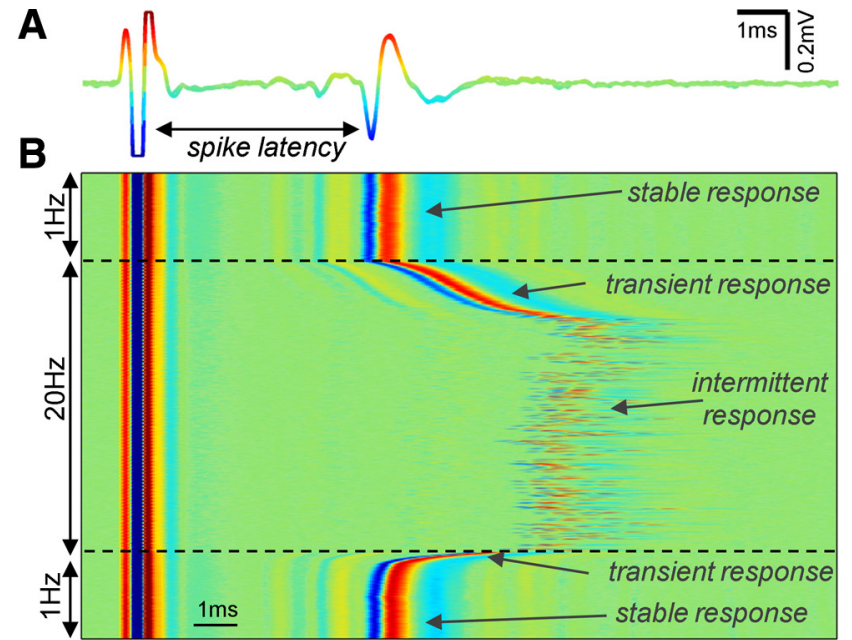

\section{C}

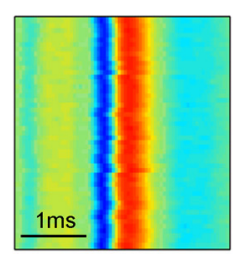

D

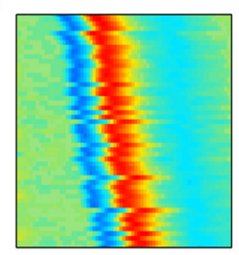

E

$\mathbf{F}$

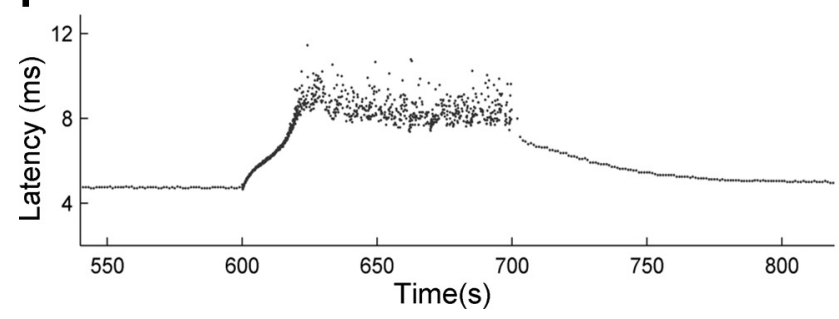

Figure 2. Responses of isolated neurons to sequences of stimulation pulses. $A$, An isolated neuron is stimulated with short ( $400 \mu \mathrm{s}$ ) electrical pulses. It responds by generating an action potential, which is recorded a few milliseconds after the stimulation. Depicted are five overlaid traces from a single neuron to pulses delivered at $1 \mathrm{~Hz}$ (interstimulus interval of $1 \mathrm{~s}$ ). Note the high temporal precision of the response. Trace is colored by voltage. $\boldsymbol{B}$, The same neuron is stimulated for $600 \mathrm{~s}$ at $1 \mathrm{~Hz}$, then $100 \mathrm{~s}$ at $20 \mathrm{~Hz}$, and again $600 \mathrm{~s}$ at $1 \mathrm{~Hz}$ stimulation. Recorded voltage traces, in response to each stimulation, are depicted using the voltage color-code shown in $\boldsymbol{A}$. The dashed lines mark points where stimulation rate is switched. Three response phases are defined, as explained in Results. Under $1 \mathrm{~Hz}$ stimulation, the response is stable and temporally precise. After the switch to $20 \mathrm{~Hz}$, a transient phase ensues, characterized by a gradual increase in spike latency. Beyond a critical latency value, the neuron enters an intermittent phase, in which spike failures occur and latency fluctuates. When stimulation rate is switched back to $1 \mathrm{~Hz}$, the neuron returns to a stable response mode after a transient recovery period. $\boldsymbol{C}-\boldsymbol{E}$, Enlargements of sections from the image in $\boldsymbol{B}$; individual spikes can be identified. Each panel is extracted from a different response phase. $\boldsymbol{F}$, A plot of spike latency as a function of stimulus time for the same data as in $\boldsymbol{B}$. For visual clarity, only $60 \mathrm{~s}$ before the first switch and 120 s after the second switch are plotted.

\section{Measurements and stimulation}

A commercial amplifier (MEA-1060-inv-BC; Multi Channel Systems) with frequency limits of $150-3000 \mathrm{~Hz}$ and a gain of $1024 \times$ was used. Rectangular $200 \mu$ s biphasic $20-50 \mu \mathrm{A}$ current or $600-800 \mathrm{mV}$ voltage stimulation through extracellular electrodes was performed using a dedicated stimulus generator (Multi Channel Systems). In the context of this study, no difference was observed in the behavior of neurons under current or voltage stimulation. Data collected through the MEAs were digitized to 16 bit using either a 5200a/526 analog-to-digital board (Microstar Laboratories) or a USB-ME256 system (Multi Channel Systems). Each recorded channel was sampled at a frequency of $20-25 \mathrm{kHz}$. One hour after the addition of synaptic blockers, the stimulation electrode was selected as the one that evoked well isolated spikes, with high signal- 
to-noise ratio, in as many recording electrodes as possible. A $25 \mathrm{~ms}$ voltage trace from those channels was collected after each stimulation pulse, and spike detection was performed offline by a manual threshold-based procedure. A 3-ms-long spike shape were extracted for each response for additional noise cleaning and analysis.

\section{Performing long-term recordings}

Whenever long-term experiments were conducted, additional criteria were applied to validate experimental stability, as discussed in the main text. Excluding the first hour, thus allowing for settling down of transient effects, spike shapes were collected, and spike amplitude distribution is created. Neurons that demonstrated SD beyond $20 \%$ of spike amplitude were excluded from the dataset. Principle component analysis (PCA) was performed on a randomly selected set of spikes (because it is impossible to perform PCA on the entire dataset, which consists of millions of spikes), and all the spikes were projected onto the first two components. The experiment was divided into three equal length segments, and spikes belonging to each of the segments were clustered together. Whenever the means of two of the three clusters were separated by more than the sum of their widths, the neuron was excluded from the dataset. Wherever more than one neuron was recorded in an experiment, cross-correlations between firing rates of the neurons were calculated. The cross-correlations were calculated by filtering the firing rate with a temporal window centered in the desired timescale and with a width equals $50 \%$ of the center value. In all the analyzed experiments, correlations were significant but small in all timescales tested (values as shown in Fig. 5 are typical).

\section{Statistical analysis of long response sequences}

For each experiment, the activity of the neuron was represented as a single point process (the recorded spike train of the neuron, without reference to stimulation time). Various statistical methods for identification of scaling phenomena and estimation of the scaling exponent were applied. Scaling exponents $\left(\alpha_{S}, \alpha_{D}, \alpha_{F}, \alpha_{A}\right)$ were estimated using leastsquares power-law curve fit to the relevant segments of the data. Although problematic in testing for power-law behavior (Clauset et al., 2009), this gives a good estimate in cases in which the power-law behavior is clear enough or when the aim is at approximating the functional relation rather than setting its exact form. Generally speaking, relations between exponent values estimated using different methods can be theoretically derived, but because the values obtained for empirical data are considerably biased, significant deviations from these relations are expected.

Periodogram (Scharf et al., 1995). A periodogram is an empirical estimator for the power spectral density of a process and is computed over the count sequence of the spike train with bin size of $1 \mathrm{~s}$. The scaling exponent $\alpha_{S}$ was estimated by fitting the low-frequency tail with a powerlaw function.

Detrended fluctuation analysis (Peng et al., 1995). Detrended fluctuation analysis (DFA) is a method designed to reveal and estimate the scaling in empirical signals. It is applicable even if the signal is strictly nonstationary. Briefly, the count sequence (with $1 \mathrm{~s}$ bins) is first integrated. A piecewise linear curve, with segments of length $T$, is fit to the data, and the mean square error (MSE) is calculated. The procedure is repeated with various values of $T$ to produce the $\operatorname{MSE}(T)$ curve. The exponent of the behavior of $\operatorname{MSE}(T)$ is an indication of scale invariance in the signal. The scaling exponent $\alpha_{D}$ was estimated by fitting the large $T$ tail with a power-law curve. An exponent value of $1>\alpha_{D}>0.5$ indicates the existence of long-range correlations, where in the limits $\alpha_{D}=0.5$ indicates uncorrelated noise and $\alpha_{D}=1$ indicates $1 / f$ noise.

Fano and Allan factors (Scharf et al., 1995; Lowen and Teich, 1996, 2005). A count sequence $Z_{T}(n)$ was calculated using logarithmically spaced bin sizes. For each count sequence, the Fano factor (variance to
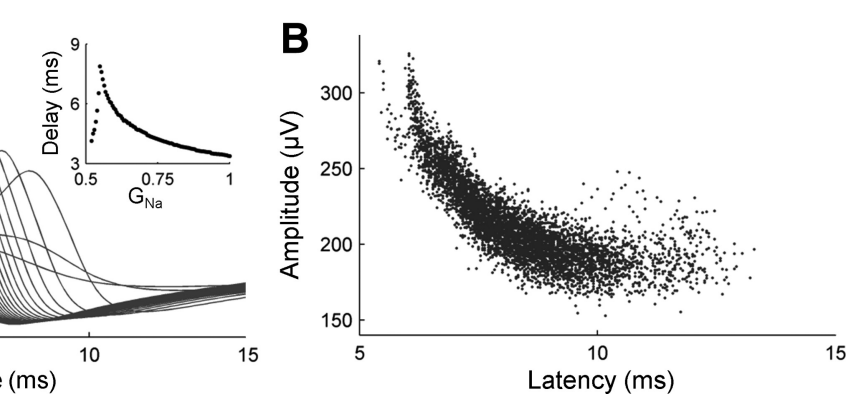

Figure 3. Spike latency and spiking probability as probes of neuronal excitability. $\boldsymbol{A}$, The effect of maximal sodium conductance The maximal sodium conductance $\left(G_{\mathrm{Na}}\right)$ was multiplied by a factor between 0.5 and 1 . As $G_{\mathrm{Na}}$ is decreased, the amplitude and olarization slope of the generated action potential are decreased, whereas the peak delay is increased. At a certain critically low oked event can no longer be considered as a propagating action potential. The inset plots the peak delay of the e as a function of the $G_{\mathrm{Na}}$ factor. $\boldsymbol{B}$, A scatter plot of the extracellular amplitude versus the extracellular spike latency from a single recorded neuron. Data are collected from the transient phases of the response to many stimulation blocks at different rates. A clear negative correlation exists between amplitude and latency.

mean ratio) is defined as $F(T)=\frac{\operatorname{var}\left(Z_{T}\right)}{\text { mean }\left(Z_{T}\right)}$ and plotted as a function of the bin size T. A variant of the Fano factor, the so-called Allan factor, is defined as $A(T)=\frac{\operatorname{mean}\left(Z_{T}(k)-Z_{T}(k+1)\right)^{2}}{2 \cdot \operatorname{mean}\left(Z_{T}\right)}$. For both factors, the scaling exponents $\alpha_{F}$ and $\alpha_{A}$ were estimated by fitting the large $T$ tails with power-law curves.

\section{Results}

\section{Spike latency and spiking probability as probes of neuronal excitability}

The main claims in this study concern the intrinsic dynamics of excitability in individual neurons, independent of synaptic and network effects. To achieve functional isolation, experiments were conducted on cultured cortical neurons, functionally isolated from their network by means of pharmacological block of both glutamatergic and GABAergic synapses (see Materials and Methods). Under these conditions, the spontaneous spiking of neurons practically stops. For 79 neurons, recorded from five different cultures, the spontaneous average spike rate was $1.12 \pm$ $0.87 \mathrm{~Hz}$ (mean $\pm \mathrm{SD}$, median of $0.52 \mathrm{~Hz}$ ) before blockers applications and $0.067 \pm 0.138 \mathrm{~Hz}$ (mean $\pm \mathrm{SD}$, median of $0.0027 \mathrm{~Hz}$ ) after the application (rates were calculated over 30 min periods, and only neurons with activity above $0.2 \mathrm{~Hz}$ before blocker application were included). With no synaptic blockers applied, neurons in the culture respond to pulse stimulation both directly and indirectly by synaptic activation. In Figure 1, the responses of a neuron to a low rate $(1 \mathrm{~Hz})$ sequence of short $(400 \mu \mathrm{s})$ pulse stimulation before and after application of blockers are compared, showing that, after the application, each stimulation evokes one spike only (presumably from a neuron that directly senses the external current) (Tal et al., 2001; Marom and Shahaf, 2002; van Pelt et al., 2004; Bakkum et al., 2008; Shahaf et al., 2008; present study), with no reverberating or synaptically mediated responses present. We have also compared the correlation between the activity levels obtained from pairs of simultaneously recorded neurons, before and after the application of synaptic blockers, over $15 \mathrm{~min}$ at high rate $(20 \mathrm{~Hz})$ stimulation. We found that blockers significantly reduce the correlation: for 18 such pairs, the correlation coefficient (calculated over $5 \mathrm{~s}$ bins) is $0.31 \pm 0.1$ (mean \pm SD absolute value), in which 11 of these 18 pairs of correlations are significant $(p<0.05)$. After the application of blockers, this correlation is reduced to $0.07 \pm 0.04$, in which none of the 18 pairs of correlation is significant. Overall, 
the lack of spontaneous activity, synaptically mediated responses, and inter-neuron correlations in the presence of synaptic blockers reassured us that there are practically no functional connections between neurons (synaptically or gap junction mediated) and that spiking activity of a given neuron reflects the interaction between the externally applied stimulation and the neuronal internal state.

Figure 2 show the dynamics of the evoked response of a single isolated neuron to a series of pulse stimulation. In Figure $2 \mathrm{~A}$, five overlaid extracellularly recorded spikes are shown; the spikes are practically identical in both shape and latency from stimulation. Note that the latency from stimulus to response is in the order of several milliseconds (van Pelt et al., 2004; Bakkum et al., 2008; Shahaf et al., 2008); this delay reflects the time it takes for the membrane to generate an action potential, as well as the conductance delay of the spike along the neurites, to the point at which it may be sensed by the recording electrode. The voltage traces of Figure $2 \mathrm{~A}$ are color coded, serving for the construction of Figure $2 B$, in which responses of the same neuron evoked by a long series of stimuli are presented. Each horizontal line of Figure $2 B$ depicts one response evoked by one stimulus; responses are ordered sequentially from top to bottom. The experiment begins with a 10 min low rate $(1 \mathrm{~Hz})$ stimulation; the response mode in this regimen is 1:1, i.e., the neuron responds to each and every stimulus (high reliability), and the trial-to-trial variability of the latency between the stimulus and the evoked spike is low (high precision). After $10 \mathrm{~min}$, the stimulation rate is increased to $20 \mathrm{~Hz}$; after this change, the response latency gradually increases while maintaining a 1:1 mode. Around a certain critical latency, the 1:1 response mode breaks down, and response failures appear in a seemingly erratic manner. Enlargements of segments, one from each phase, from the image of Figure $2 B$ are shown in Figure $2 C-E$, allowing identification of individual spikes. Note that the response latency beyond the critical point fluctuates around a (more or less) constant value. After $100 \mathrm{~s}$, the stimulation rate is decreased back to $1 \mathrm{~Hz}$, and both latency and response probability gradually recover. Figure $2 F$ shows the latency dynamics throughout the experiment using a real-time (rather than stimulus index) axis. The phenomena of latency increase and spike failures are by themselves not new and have been observed intracellularly in the cortical culture preparation used here (Tal et al., 2001), as well as in several other preparations both extracellularly and intracellularly (Spira et al., 1976; Grossman et al., 1979; Aston-Jones et al., 1980; De Col et al., 2008; Scroggs, 2008; Ballo and Bucher, 2009); as explained below, here we use these two extracellularly obtained response features (latency and failure) as probes in the study of neuronal excitability dynamics.

In general, neuronal excitability can be defined as the relation between exciting and restoring forces; in the most simple neuronal setting, this relation translates into the ratio between sodium and potassium conductances, as in the canonic Hodgkin-Huxley $(\mathrm{HH})$ model. Figure $3 \mathrm{~A}$ shows a simulated realization of an $\mathrm{HH}$ model, in which the maximal sodium conductance, $G_{\mathrm{Na}}$, is systematically varied. A short $(400 \mu \mathrm{s})$ current pulse is applied to the model membrane, generating an action potential. As $G_{\mathrm{Na}}$ is lowered, the recruitment time of the spike is substantially increased, causing a delayed response (Fig. $3 A$, inset). This monotonic inverse relation between excitability and stimulus-spike delay is further extended when the spike propagates along a neurite, i.e., propagation velocity decreases and delay is accumulated (De Col et al., 2008), a likely explanation for the relatively high values and wide range of delays evident in Figure 2. When excitability drops below a certain critical level, the evoked response is expected to
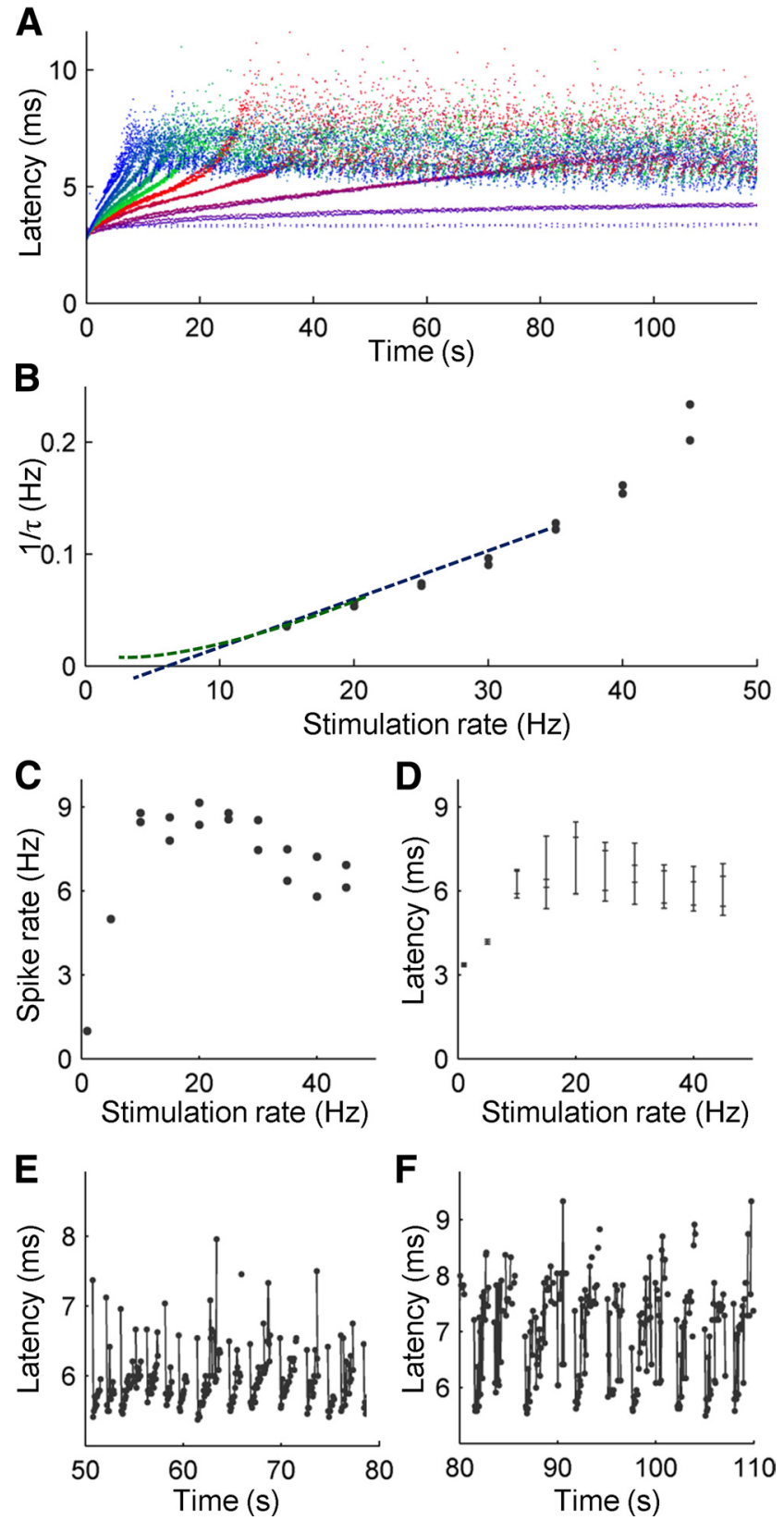

Figure 4. The dependency of neuronal response on stimulation rate. A neuron was stimulated at rates of $1,5,10,15,20,25,30,35,40$, and $45 \mathrm{~Hz}$. Stimulation was delivered in blocks of $120 \mathrm{~s}$ duration. The neuron was allowed to recover for $5 \mathrm{~min}$ between each block. Blocks of different rates were delivered randomly. Two blocks of each stimulation rate were delivered. $\boldsymbol{A}$, Spike latency as a function of time from block onset. Each color designates a different stimulation rate. Note that the higher the stimulation rate, the faster the approach to the critical latency. $\boldsymbol{B}$, The reciprocal of the timescale of the transient phase (defined as halftime to reach the critical latency) is plotted as a function of stimulation rate. Two possible scenarios for extrapolation to low stimulation rates are considered, as explained in Results. The first, depicted blue, is a linear back extrapolation to a critical stimulation rate below which intermittency is not reached. The second back extrapolation, depicted green, allows for the possibility that transition to intermittency occurs, regardless of stimulation rate, given enough time. $\boldsymbol{C}$, A plot of mean spike rate estimated from the last 30 s of each block, as a function of stimulation rate. Note that firing rate remains more or less stable beyond $\sim 10 \mathrm{~Hz}$ (albeit a minor drop at the very high stimulation rates). $\boldsymbol{D}$, A plot of latency in the last $30 \mathrm{~s}$ of each block (mean $\pm \mathrm{SD}$ ) as a function of stimulation rate. Latency seems to be unaffected by stimulation rate in the intermittent phase. $\boldsymbol{E}, \boldsymbol{F}$, Two extracts, from a $15 \mathrm{~Hz}$ block $(\boldsymbol{E})$ and a $20 \mathrm{~Hz}$ block $(\boldsymbol{F})$, showing the complex fluctuation of the spike latency around its mean. The lines connect consecutive responses (that are not separated by failures). 
attenuate quickly and propagation is aborted, thus setting an effective spikegeneration threshold on membrane excitability under given stimulation amplitude.

Although in real neurons things can be much more complicated because excitability is affected by many membrane currents and cellular factors, it is reasonable to assume that these affect spike latency and spiking probability in a similar way. Note that excitability substantially affects not only the latency but also the slope (i.e., derivative) of the voltage at the depolarization phase of the spike (Fig. $3 A$ ). Thus, a correlation is expected to be found between extracellularly recorded spike amplitude and spike latency. Figure $3 B$ shows a scatter of spike amplitudes versus spike latencies, collected from a single neuron, verifying this expectation in the extracellular measurements. Finally, supplemental Figure S1 (available at www.jneurosci. org as supplemental material) shows that the above-mentioned increase of spike latency and decrease of spike amplitude and depolarization slope can be observed using intracellular voltage recording in response to high rate sequence of stimuli. Together, the above considerations and observations strengthen our interpretation of extracellular measures of spike latency and spiking probability as indirect probes of neuronal excitability.

\section{Neuronal response to ongoing stimulation is characterized by distinct phases}

We denote the different phases seen in Figure $2 B$ as stable, transient, and intermittent (depicted). To characterize the dependence of the above phases on stimulation rate, we stimulated neurons using blocks of different rates, each lasting $120 \mathrm{~s}$. The neuron was allowed to recover for $5 \mathrm{~min}$ between blocks. Blocks of different stimulation rates were randomly ordered, and each rate was repeated twice. Figure $4 A$ shows an example of such an experiment in one neuron. In the 1 and $5 \mathrm{~Hz}$ blocks, after a transient phase, the latency seems to stabilize below the critical latency value (De Col et al., 2008), and the response mode is 1:1 throughout. Above $5 \mathrm{~Hz}$, the critical latency is reached and the neuron enters the intermittent phase. Note that repeats of the same stimulation rate leads to highly similar trajectories in the transient phase, as well as similar critical latencies.

Figure $4 B$ shows the timescale of the transient phase leading to the critical point, as a function of stimulation rate. This relation is clearly monotonic and (by extrapolation) suggests the existence of a critical stimulation rate, somewhere at $\sim 5 \mathrm{~Hz}$, below which the neuron does not enter the intermittent phase (depicted by a blue dashed line). In this scenario, the time it takes to reach the critical latency diverges as the critical stimulation rate is approached. Of course, one cannot rule out, in a finite experimental setting, the possibility that there might be an extremely slow transition to the intermittent phase even below $5 \mathrm{~Hz}$ (depicted with a green dashed line). In 21 different neurons the mean extrapolated critical stimulation rate was $7.92 \pm 4.7 \mathrm{~Hz}$, with values ranging from as low as $\sim 2 \mathrm{~Hz}$ to as high as $\sim 23 \mathrm{~Hz}$. Unlike the timescale of the transient phase, the value of the critical latency (estimated

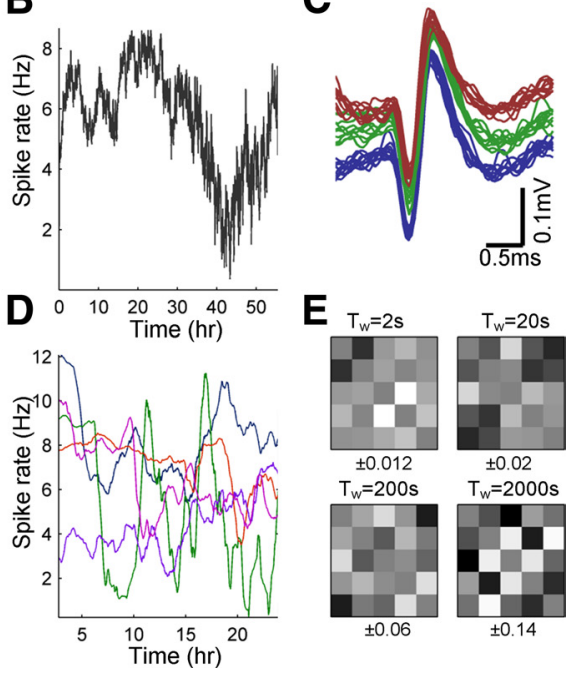

c

Figure 5. Performing long recordings from isolated neurons. $A$, A neuron was stimulated for $55 \mathrm{~h}$ with a continuous series of

B
$120 \mathrm{~s}$

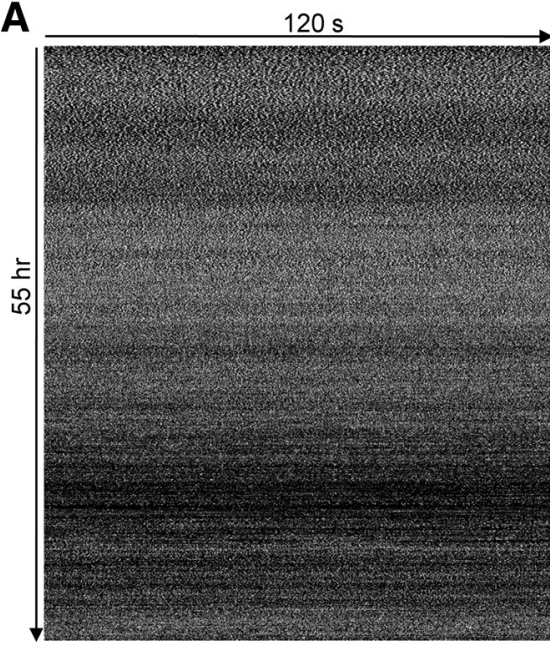
pulses delivered at $25 \mathrm{~Hz}$. Depicted is the binary response sequence (a white pixel represents a spiking response, and a black pixel (The sequence is wrapped at 2 min duration (start of experiment is at top left corner). The most $\boldsymbol{E}$, Cross-correlation between the firing rates from $\boldsymbol{D}$, computed for different timescales (see Materials and Methods). The range below each panel designate the full range of values for each matrix.

as the mean latency during the intermittent phase) is independent of the stimulation rate. This is seen in Figure $4 D$ for one given neuron. The temporal structure of the fluctuation of spike latency around the mean is not structureless (Fig. 4E,F) and probably reflects the underlying complex processes. Similar results were obtained for the relation between stimulation rate and firing rate (Fig. 4C), in which after the intermittent phase is reached, no increase in firing rate (as estimated from the spike count in the last $30 \mathrm{~s}$ of each block) is observed for higher stimulation rates. The results of Figure $4 D$ imply an intrinsic limit to the firing rate of neurons when averaged over a sufficiently long time. Over 21 analyzed neurons, the thus defined maximal rate was found to be $8.24 \pm 4.2 \mathrm{~Hz}$.

Because the experiments reported here were conducted extracellularly on synaptically blocked cultured neurons, it is fair to ask the following: do the phenomena observed here reflect the special conditions of the cultured preparation and the limitations of the extracellular electrical interface? Supplemental Figure S1 (available at www.jneurosci.org as supplemental material) shows that the three phases reported above (stable, transient, and intermittent), as well as the existence of critical stimulation frequency, can also be observed using intracellular recordings. Intracellular methods, however, cannot provide the length of stable recordings necessary for many of analyses performed above and those that will follow.

In which of the three phases, depicted in Figure $2 B$, resides a cortical neuron during ongoing activity, in its natural setting? The answer to this question has cardinal consequences in the interpretation of activity fluctuation in vivo. There are many reasons, experimental observations, and theoretical considerations to believe that most neurons reside in the intermittent phase, in which excitability fluctuates around a critical value for spike generation. Taking into account cortical connectivity, typical fir- 

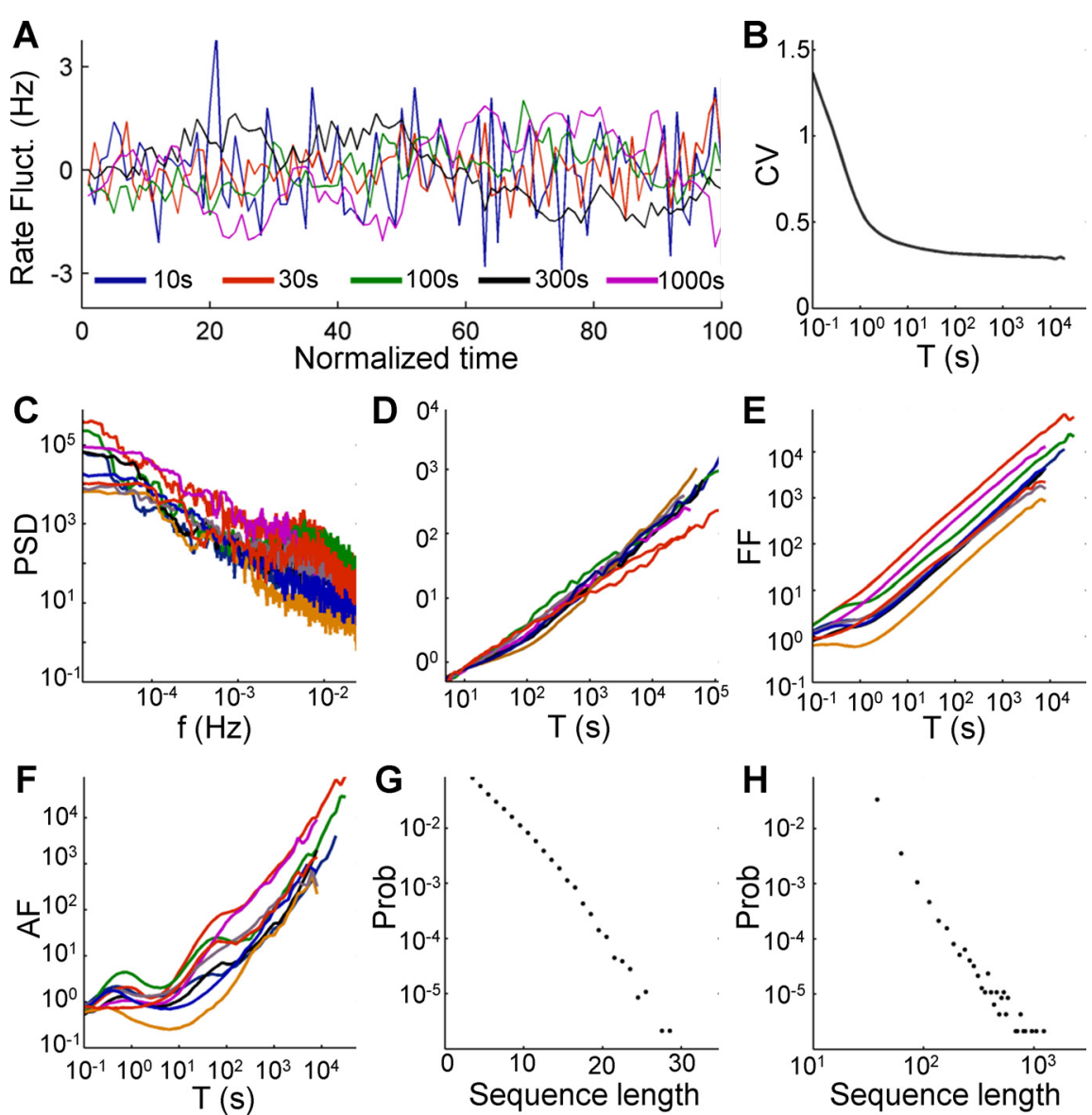

Figure 6. The rate dynamics in the intermittent phase is scale free. $\boldsymbol{A}$, The firing rare fluctuations of the neuron from Figure $3 A$. Firing rate is estimated using bins of different sizes $(10,30,100,300$, and $1000 \mathrm{~s})$ and plotted on normalized time axis (units in number of bins), after subtracting the mean of each series. Although the bin duration is increased by two orders of magnitude, the overall variability in the rate does not reduce significantly. $B, C V$ of the bin counts, as a function of bin size, plotted on a log-linear axis. After an initial decrease in the $\mathrm{CV}$, it is practically stabilized above 0.3 . The $\mathrm{CV}$ of a signal with finite correlation length is expected to decrease as $T^{-0.5}$. C, Firing rate periodograms of nine neurons stimulated for $24-96 \mathrm{~h}$ with stimulation rates of $20-25$ $\mathrm{Hz}$. Significant structure can be identified in the higher frequency regimen, but $1 / f$-type behavior is abundant in the low-frequency tails for all the neurons. $\boldsymbol{D}$, DFA for the same nine recorded neurons, showing power-law increase in root mean square (RMS) as $T$ increases. $\boldsymbol{E}$, Fano factor (FF) curves for the nine recorded neurons. $\boldsymbol{F}$, Allan factor (AF) curves for the nine recorded neurons. $\boldsymbol{G}$, Length distribution of spike-response sequences, on a half-logarithmic axes, demonstrating an exponential behavior. $\boldsymbol{H}$, Length distribution of no-spike-response sequences, on a double-logarithmic axes, demonstrating a power-law-like behavior.

term statistics. We confine our analyses of the intermittent phase to the neuronal rate dynamics rather than spike latency. Rate is a continuous process, constructed from a point process, for which analyses tools and methods are abundant and thoroughly discussed in the literature. We leave the analysis of spike latency series, which is a more generalized form of a time series (and indeed more difficult to analyze), to future investigation.

\section{Resolving dynamical from experimental instabilities in the intermittent phase}

Stimulating a neuron in a rate that exceeds the critical value for long periods of time (hours, days) enables exploration of neuronal excitability dynamics over littlestudied, yet highly relevant, timescales. Figure $5 A$ shows an example for such a temporally extended experiment: a neuron was stimulated continuously for $55 \mathrm{~h}$ at $25 \mathrm{~Hz}$. The most prominent feature seen in Figure $5 A$ is the significant modulations in firing rate over many timescales (Fig. 5B), up to the timescale of the experiment itself, in an apparently nonstationary manner (i.e., rate statistics such as the mean, variance, and autocorrelation seem to change during the experiment).

Analysis of slowly fluctuating responses in such long experiments calls for careful measures to exclude sources of variability that arise from experimental instability. The primary measure we used is the stability of spike shape and amplitude throughout the experiment, both sensitive to cell vitality as well as to the relative position of the cell and recording electrode. Only neurons that complied

ing rates, membrane time constant, and threshold potential (Abeles, 1991), a conservative estimation of suprathreshold event rate may be obtained; this estimate turns out to be $>10 \mathrm{~Hz}$, exceeding the typical firing rate of a cortical neuron. Indeed, intracellular measurements from cortical neurons conducted over the past 40 years support this estimation, showing that the rate at which neurons are excited "above threshold," regardless of how threshold is defined, can be up to an order of magnitude larger than their actual firing rate (Elul and Adey, 1966; Heck et al., 1993; Carandini, 2004). This gap is commonly explained by considering a probabilistic threshold function, in which the likelihood for generating a spike is dependent on instantaneous membrane potential and maybe on the elapsed time from the last generated spike (the popular renewal process model). However, experimental evidence suggest (Heck et al., 1993; Carandini, 2004) that a significant contribution for the above gap comes from membrane dynamics, reflecting slower modulating processes.

In what follows, we focus on the dynamics within the intermittent phase, including its detailed patterns and long- with strict spike shape stability criteria (see Materials and Methods) were considered in the analyses that are presented below. Figure $5 C$ demonstrates the stability of spike shape and amplitude during the experiment of Figure 5A. Stable spike shape, however, is not sufficient to exclude external modulations of neuronal activity arising from drifts and fluctuations in experimental environment conditions (e.g., glucose concentration, $\mathrm{pH}$, temperature, electrode properties), which are more difficult to control and monitor. We therefore adopted a practical approach, relying on an assumption that such global environmental factors are likely to have a similar effect on all the neurons in the culture: during an experiment performed on a given culture, we simultaneously recorded from a number of neurons that independently respond to the same stimulus series (see Material and Methods). Figure $5 D$ shows examples of spike rate fluctuations in five neurons that were recorded simultaneously over $24 \mathrm{~h}$ under continuous stimulation at $20 \mathrm{~Hz}$. The correlations between the activities of these neurons can serve as a measure for instability of responsiveness attributable to unstable environmental (global) conditions. To separate between contributing effects of different 
timescales, we filtered the firing rate with temporal windows of various durations. Figure $5 E$ shows an example for such an analysis, in which correlation coefficients between the activity of five neurons, recorded from the same culture, are computed. These correlations are relatively low (up to $\sim 0.1$ ) for all window durations (a slight increase in correlation is observed for the slower timescales, which may result from the wider temporal window used, allowing for more independent sources of variability; see Materials and Methods). Moreover, the coefficients appear pairwise (rather than global), suggesting that they are not induced by global experimental fluctuations. The uncorrelated variability, together with the stable spike shape and spike amplitude, allowed us to assume that the instability of neuronal responsiveness of the kind seen in Figure $5 A$ reflects dynamical instability that is intrinsic to the neuron itself. In this study, we summarize results from nine neurons, recorded from three cultures; spike shape, spike amplitude, and cross-correlation analyses were used to verify stability in all these experiments (see Materials and Methods).

\section{Scale-invariant rate dynamics in the intermittent phase}

The firing rate of the neuron in the experiment of Figure $5 A$, plotted in Figure $5 B$, is highly variable and seems to fluctuate on many timescales. To gain insight into the statistical nature of these fluctuations, we compared the firing rate on multiple scales by computing the rate using different bin sizes and plotting the estimate on a normalized time axis (Fig. 6A). A signal with short correlation length (e.g., low-pass filtered white noise or renewal process with exponential decaying interval distributions) is expected to exhibit reduced rate variability with a larger bin; at the limit of large time bins $(T)$, the SD $(\sigma)$ should decrease as the inverse of the time bin used. In contrast, the plot of Figure $6 \mathrm{~A}$ reveals scale invariance (or, more accurately, statistical self-similarity): the rate seems to fluctuate to the same extent in all the scales used and to follow similar statistics. This can be demonstrated by plotting the coefficient of variation $(\mathrm{CV}=\sigma / \mu)$ of the spike count histogram as a function of the bin size (Fig. 6B): although it does decrease for small $T$ (up to a scale of seconds), the decrease rate becomes extremely slow on larger $T$ values and seems to stabilize above $\sim 0.3$.

The scale invariance, or self-similarity, of a time series can be analyzed using various measures and statistics, all intended at estimation of a scaling exponent $\alpha$ (Bassingthwaighte et al., 1994; Beran, 1994; Scharf et al., 1995; Robinson, 2003; Lowen and Teich, 2005). The value of this exponent provides a measure of the degree of irregularity of the sequence of spikes that is preserved (rather than averaged out) over different extended timescales. It can provide important information with regard to the type of process or mechanism underlying the scale-invariant behavior. Choosing a proper estimation method for the scaling exponent, however, is a delicate matter, requiring certain assumptions to be made about the nature of the signal at hand (Taqqu et al., 1995; Esposti et al., 2008). Here we used several methods, all showing that scaling exists; however, we refrain from basing specific claims on the actual values of the scaling exponent. The most intuitive measure used here is the periodogram, an empirical power spectrum density estimator. Figure $6 C$ shows the periodograms of nine recorded neurons on a double-logarithmic axes, revealing a power-law-like behavior over a considerable range. This $1 / f$-type power spectrum is a signature of self-similar processes and can be used for the estimation of the scaling exponent by fitting a power-law curve to the low-frequency tail (Scharf et al., 1995; Lowen and Teich, 1996); the resulting values obtained by such a fit are $\alpha_{S}=1.43 \pm 0.35$ (mean \pm SD) for all
A

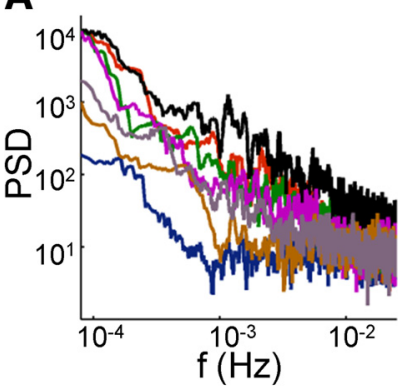

B
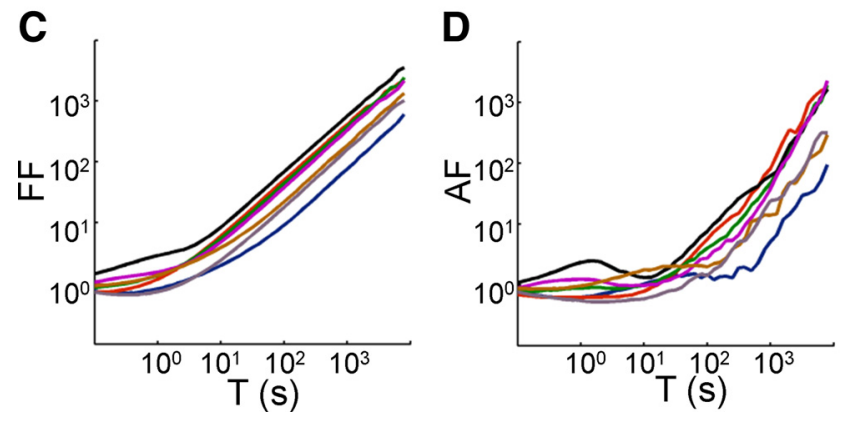

Figure 7. Intermittency under stochastic stimulation. Neurons were stimulated with a Poisson stochastic stimulation sequence, created by drawing intervals from an exponential distribution, and the scaling analysis of Figure 6 was repeated. $\boldsymbol{A}-\boldsymbol{D}$ show, for seven neurons stimulated at $20 \mathrm{~Hz}$, that the periodogram DFA, Fano factor (FF), and Allan factor (AF) analyses retain their characteristic power-law shape, similar to the ones obtained by constant interstimulus intervals.

the recorded neurons. A related measure often used for the identification of scale invariance in time series is DFA, which is also applicable in cases in which the time series is formally nonstationary (Peng et al., 1995). DFA curves for nine measured neurons are plotted in Figure $6 D$, showing a power-law behavior with a scaling exponent of $\alpha_{D}=0.94 \pm 0.28$. Other measures and methods for revealing scaling phenomena, such as Fano factor and Allan factor (Scharf et al., 1995; Lowen and Teich, 1996, 2005), exhibit a similar relation (Fig. 6E,F). Note that, although the structure, evident in specific bands (for example, in the periodogram and Allan factor analyses of Fig. 6C,F), implies the existence of underlying modulating processes with uniquely defined timescales, the power-law behavior in the slow tail is observed in practically all neurons. The analyzed neurons also differ by a variety of multiplicative factors, expressing the vertical offsets observed in the periodogram (Fig. 6C), Fano factor (Fig. 6E), and Allan factor (Fig. 6 F). These multiplicative factors reflect the total variability (or power) of the signal rather than its temporal structure.

When experiments of limited duration are conducted, neuronal responses to a stochastic input (usually white Gaussian noise) are shown to be more precise and reliable then responses to constant input (Mainen and Sejnowski, 1995). With this in mind, we repeated the analyses of Figure 6 using a white stochastic input, namely a Poisson process, yielding similar qualitative results (Fig. 7 ), indicating that, in contrast to responses of neurons in shortterm experiments, when long-term features are considered, the variability in response to white noise is the same as the variability in response to constant-frequency stimulation.

To gain additional insight into the features of the spike trains underlying the scale-invariant behavior, we divided the response time series to binary sequences of uninterrupted consecutive spikes (" 1 ") and sequences of uninterrupted consecutive failures 


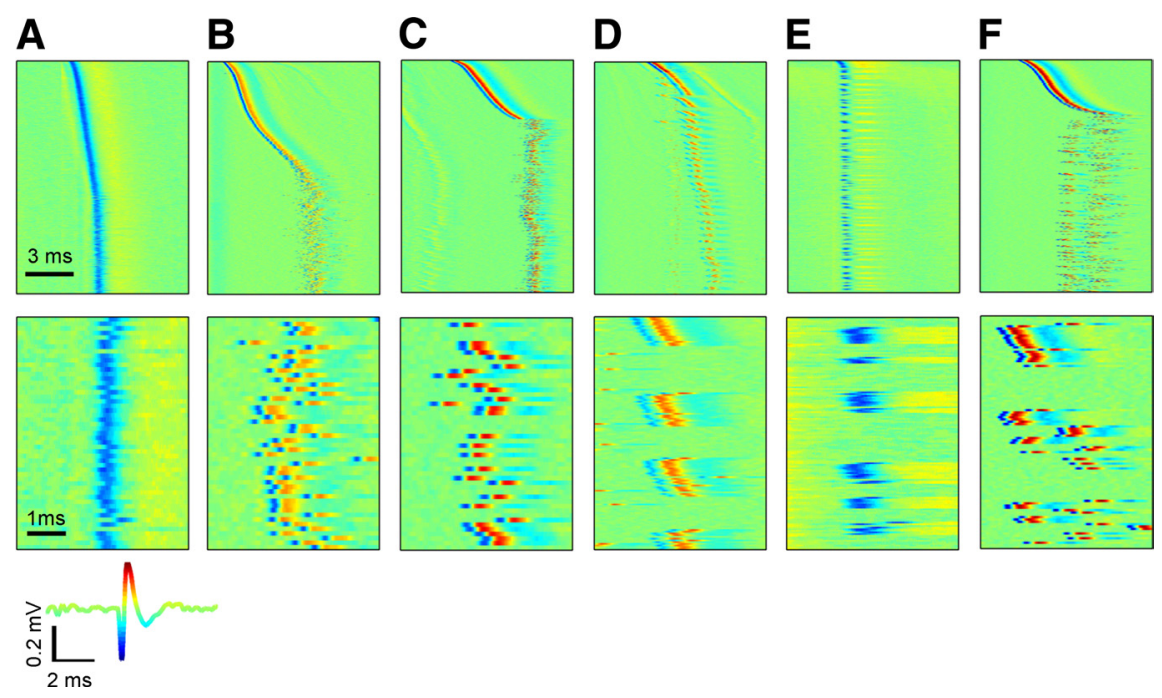

Figure 8. Prototypic onset response patterns. $\boldsymbol{A}-\boldsymbol{F}$ show the response of six different neurons to the onset of a $20 \mathrm{~Hz}$ stimulation block. The top panels show the entire response to a 2 min stimulation block; the bottom panels show an enlargement of a segment within the intermittent phase, allowing for identification of specific response patterns. All panels share the same color scale, as presented in the colored spike (bottom left). $A$, A highly reliable neuron, responding with a low-jettered latency. This neuron is well below its critical stimulation even at $20 \mathrm{~Hz}$ stimulation rate. $\boldsymbol{B}$, A reliably responding neuron: this neuron is stimulated just below its critical stimulation rate. In this case, although the latency does fluctuate considerably, systematic failures do not appear, and the neuron responds to practically all delivered stimuli. $C$, An irregularly responding neuron; spikes and failures appear in a seemingly random manner. D, Bursting-like neuron; spikes are clustered together in a highly ordered manner, with typical cluster size and intercluster intervals. $\boldsymbol{E}$, A neuron responding in a clustered manner, with irregular cluster sizes and intervals. $\boldsymbol{F}$, A neuron responding with complex patterns of clusters and individual spikes. Latency tends to jump between two levels.

("0"). Histograms of the lengths of these sequences are shown in Figure 6, $G$ and $H$, for the experiment of Figure $5 A$. Interestingly, there is a considerable difference between the histograms, in which the 1 sequence lengths (which can be thought of as "burst lengths") are distributed exponentially (Fig. 6G), whereas the 0 sequence lengths ("interburst intervals") are power-law distributed (or, cautiously, heavy-tailed) (Clauset et al., 2009). This means that the response is dominated by long "breaks" in which the neuron is unresponsive. The qualitative difference in the histograms implies that rate modulation is not sufficient to explain the observed dynamics and additional structure exists in the response sequence. It should be noted, however, that although power-law distributed intervals can lead to scaleinvariant behavior in the form of a fractal renewal process (Barabási, 2005; Lowen and Teich, 2005), in this case the intervals are not independent identically distributed, as can be demonstrated for example by plotting the interval sequence correlogram (data not shown) or by mere observation of repeatable patterns (see next section). Thus, the resulting power-law distribution seems to reflect the aggregation of many local exponential distributions (i.e., estimating this distribution over short timescales will result in an exponential-tailed distribution) rather than a stationary power-law distribution (Marom, 2010).

\section{The intermittent phase is characterized by quasi-stable pattern modes}

Neurons are often classified by the activity patterns they exhibit in response to input (Markram et al., 2004). In most of these classification studies, the neuron is stimulated intracellularly by a current step after a "reset" period in which no input is applied. In analogy to these patterns, we identify prototypical behaviors in the response of isolated neurons to a series of high rate extracellular pulse sequences. Figure $8 A-D$ shows four examples for response patterns exhibited by different neurons in response to the onset of a $20 \mathrm{~Hz}$ stimulation block. "Classes" of response, thus viewed, include, for example, stable response, irregular response, regular-clustered responses, and irregular-clustered responses and might mirror neuronal classes as observed with intracellular stimulation: regular and irregular spiking, bursting, and stuttering (more elaborate distinctions might be possible with additional refined experiments). Extending this analogy, the gradual increase in spike latency observed in the transient phases, as well as within uninterrupted consecutive evoked spikes (as can be seen, for example, in Figs. $4 E$, $8 D, F)$ resembles spike rate adaptation; in the latter, the decrease in excitability is manifested by increased interspike intervals rather than by an increase in stimulus-spike latency. The different stimulation protocols render different response dynamics, thus making mapping of concepts difficult. However, it is reasonable to hypothesize that the mechanisms underlying the patterns produced by these different protocols are related to each other. The response pattern of a given neuron to the onset of a stimulation block can be dependent on the stimulation rate applied but remains robust over repetitions of blocks with the same stimulation rate (Fig. 9).

However, are these patterns and classes stable throughout? If not, their usefulness for functional classification of neurons might be limited. When the fine structure of long spike trains that are evoked as described in the previous section is carefully examined, distinct modes of activity are identified, each characterized by a typical pattern. Figure 10 shows several examples for such modes, extracted from the intermittent response of a single neuron to a long series of a constant-frequency stimulation. Although such a mode is typically stable for minutes and more, when observed over extended duration, the response of the neuron undergoes transitions between these quasi-stable modes in an unpredictable and nonstationary manner. These transitions between modes can be sharp and instantaneous or sometimes gradual. In fact, the range of different patterns exhibited by a given neuron can be sometimes as wide as the range of patterns exhibited by the entire neuronal population over a temporally bounded observation window. This observation casts doubt on the validity of extrapolating from the behavior of a neuron in one given timescale to its continuous, long-term behavior.

\section{Discussion}

We studied here the dynamics of neuronal excitability over an extremely wide range of timescales (milliseconds to days) in cultured cortical neurons, using extracellular electrophysiological means, applying long series of stimulation pulses within a physiologically relevant range of rates. We identified three distinct phases of neuronal responses to such stimulation: a stable phase, usually observed under low stimulation rates (below $\sim 5 \mathrm{~Hz}$ ), in which response is reliable and its latency is precise; a transient phase, observed after a switch in stimulation rate, in which response reliability and latency undergo a gradual change; and an intermittent phase, observed under high stimulation rates, in which response failures appear in a seemingly erratic manner and 

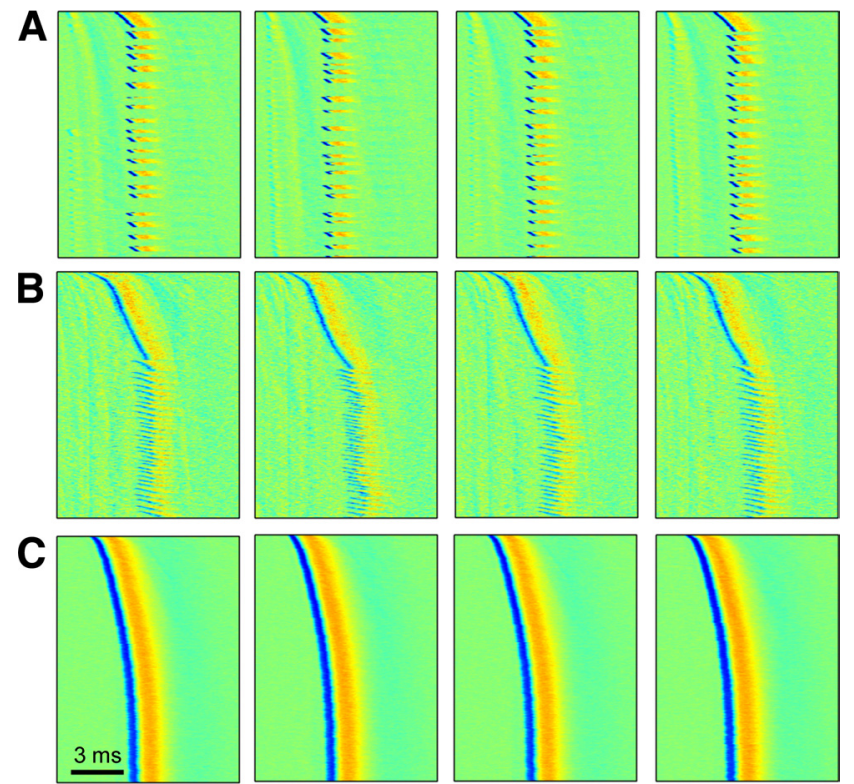

Figure 9. The repeatability of isolated neurons response patterns. Neurons are stimulated for $1 \mathrm{~h}$ with a series of $20 \mathrm{~Hz}$ stimulation blocks, each lasting 2 min and separated by $3 \mathrm{~min}$ from the previous block. The responses of three neurons $(\boldsymbol{A}-\boldsymbol{C})$ to four of the stimulation blocks are shown. Panels share the color scale of Figure 8. Although repetitions might defer in the fine details (e.g., oscillation phase), the response pattern types exhibited by the neurons are stable throughout the experiment.
A

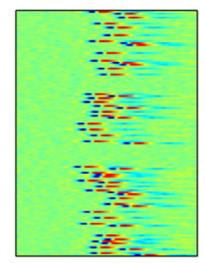

E

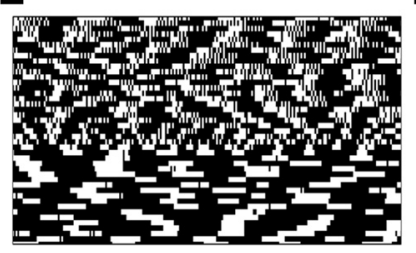

C

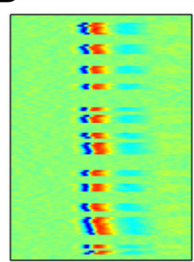

$F$

$F$
D
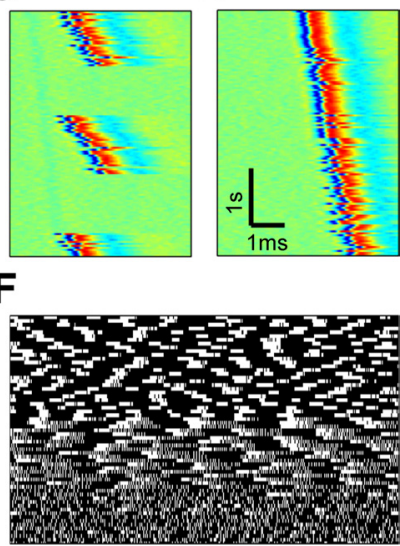

Figure 10. Quasi-stable pattern modes in the intermittent phase. Many of the recorded neurons undergo transitions between response pattern modes during the intermittent phase, on top of rate modulations. $\boldsymbol{A}-\boldsymbol{D}$ show examples for such modes, in one given neuron, recorded over a long period of time under $25 \mathrm{~Hz}$ stimulation. All panels show data segments of $5 \mathrm{~s}$ (125 responses) and share the same color scale of Figure 8. Typically, a quasi-stable mode lasts for several minutes. $\boldsymbol{A}$, An irregular mode, in which spikes and failures appear in a disordered manner, and latency fluctuates considerably. $\boldsymbol{B}$, Short bursting mode, in which spikes appear in small clusters. Cluster sizes and the intervals between them are relatively variable, and spike latency is relatively stable. $\boldsymbol{C}$, Long bursting mode, in which cluster sizes are significantly larger. Cluster sizes and intercluster intervals are relatively stable (a feature that cannot be detected in this figure because of presented short duration). Note latency dynamics within each cluster. $\boldsymbol{D}$, A long response mode, in which consecutive spiking occur over few tens of seconds. More often than not, such a mode is seen after a relatively long break. $\boldsymbol{E}, \boldsymbol{F}$, Examples of binary response matrices, as in Figure $3 A$, of the response around mode transitions. $\boldsymbol{E}$ begins with a mode in which clusters of spikes, each of which slowly decays into complete quiescence, abruptly change to a mode in which clusters end sharply. $\boldsymbol{F}$ shows a more gradual transition between two modes of responses, from a clustered response mode, through an intermediate region, to an irregular response mode.

latency fluctuates around a constant value. Our data indicates that the transition to intermittency is characterized by a critical latency (hence a critically low excitability) that, whenever reached, is followed by large fluctuations in both latency and responsiveness. Our data also imply an existence of a critical stimulation rate beyond which a transition to the intermittent phase will occur. Once intermittency is reached, response failures maintain the mean firing rate $\sim 7-10 \mathrm{~Hz}$, independent of stimulation rate, reflecting a possible intrinsic limit to the firing rate of neurons when estimated over long periods. It is important to note that usually the bounded firing rate of cortical neurons in vivo is attributed to network effects (e.g., balanced inhibition); our results point to an "intrinsic" limit to firing rate. Likewise, the stimulus-response latency during the intermittent phase fluctuates around a mean value that is independent of stimulation rate, possibly indicating that neuronal excitability is fluctuating around the critical spiking threshold.

We have shown that, within the intermittent phase, the response of isolated neurons follow complex dynamics. The statistics of this response point to underlying processes that are practically timescale invariant, i.e., not characterized by a discrete and finite set of time constants; rather, there seems to be a continuum that covers any observable scale. We demonstrated this point by various statistical measures, such as the periodogram (Scharf et al., 1995; Lowen and Teich, 1996), detrended fluctuation analysis (Peng et al., 1995), and Fano and Allan factors (Lowen and Teich, 1996, 2005). Moreover, durations of nonresponsiveness follow a power-low distribution, whereas durations of consecutive responses are exponentially distributed, suggesting that the nature of the underlying forces differ; interestingly, an analogous asymmetry is found in the behavior of the voltage-dependent sodium channels, one of the principle determinants of excitability in these neurons (Marom, 2009; Soudry and Meir, 2010). Altogether, these analyses reveal the long-range interdependencies and slow modulations that characterize neuronal activity when observed over extended durations.

An important implication of the above results is that the gap between input and output rates seen in our data, as well as in data obtained in vivo, is not describable in terms of renewal process models, in which the likelihood of spike generation is a function of instantaneous membrane potential and the time elapsed from the last spike. Rather, the temporal distribution of spike failures seems to reflect complex underlying dynamics of membrane excitability and spiking threshold.

We have also demonstrated how the classes and types of neuronal electrophysiological activity, as often identified by intracellular current injection and measurements (Markram et al., 2004), are mirrored in our extracellular paradigm. Furthermore, we have shown that, when observed continuously, the response of neurons is characterized by quasi-stable modes, each identified with a typical response pattern. A single neuron, when observed over long enough duration, can exhibit a rich repertoire of response patterns; furthermore, the response to the onset of stimulation cannot be taken as an indicator of the ongoing response.

The set of observations reported here at the single-neuron level were obtained using stimulation parameters that are not much different from what a cortical neuron experiences in vivo. Our results show that, under these conditions, neuronal excitability resides near a critical point, characterized by intermittency, critical correlated fluctuations, and quasi-stable pattern modes, maintained by underlying activity-dependent processes. There are reasons to believe that this might also be the situation in vivo (Elul and Adey, 1966; Heck et al., 1993; Carandini, 2004). The immensity of the cellular processes involved in modulating excitability over long timescales suggests that a high-dimensional or stochastic model (Englitz et al., 2008) is required to capture the observed complexity. However, it is tempting to speculate that 
the long-term neuronal behavior is describable in terms of a low dimensional nonlinear dynamical process (Selz and Mandell, 1992). Hopefully, future experiments and analyses might distinguish between possible generative models that are capable of reproducing the observed set of phenomena, leading to a construction of a mathematical object that faithfully represents neuronal excitability over long timescales.

All of the observations in this study were drawn from experiments on isolated neurons, thus exposing intrinsic excitability dynamics. It is clear, however, that in natural settings, in both culture and the brain, these dynamical properties are coupled to (and modulated by) other processes, at different levels of organization. These can include synaptic dynamics, neuromodulation, population dynamics, and many more. Nevertheless, whenever long-enough experiments are performed and intertrial dynamics are analyzed, temporal complexity appears independent of the level of organization and the measured signal (for a recent review, see Marom, 2010). This is the case in psychophysical, behavioral, and cognitive experiments (Anderson, 1982; Rose and Lowe, 1982; Magnussen and Greenlee, 1985; Greenlee and Magnussen, 1987; Logan, 1988; Chen et al., 1997; Gilden, 2001; Newell et al., 2001; Monto et al., 2008), as well as in measurements obtained using functional magnetic resonance imaging, magnetoencephalography, electroencephalogram, local field potential, and spike trains recorded from network-embedded neurons in vivo and in vitro (Teich, 1989; Teich et al., 1997; Lowen et al., 1997; Linkenkaer-Hansen et al., 2001, 2005; Buzsaki, 2006; Nir et al., 2007, 2008; Monto et al., 2008; Wink et al., 2008; Esposti et al., 2009; Freyer et al., 2009). Complexity is preserved even at the level of single functional proteins, the ionic channels underlying the phenomenon of excitability, including scale-free dynamics bounded only by experimental limitations (Liebovitch et al., 1987; Toib et al., 1998; Ellerkmann et al., 2001). Moreover, many of the complex features described here are not unique to neural excitability and are observed in other biological excitable systems as well (Soen and Braun, 2000).

This being the case, it is difficult to turn a blind eye at the opportunistic choices made by us, physiologists, and our fellow theorists as to what should or should not be included in neural models in general and models of neuronal excitability in particular. We do have a tendency, when analyzing a given level of organization, to attribute the observed complexity to extended spatial constraints at that given level; complexities at the levels below are assumed to average out or to be negligible. However, when complexity goes all the way down to the molecular level, we can no longer allow ourselves to construct models of brain function using arbitrary cutoffs that are based on a temporal hierarchy: brain phenomena extend throughout the range of milliseconds, seconds, to days, and beyond; single neurons also have, in principle, the arsenal of machineries to contribute and modulate their electrical activity over milliseconds, seconds, to days, and beyond. Hence, models of excitability should account for that extended range and must be considered when modeling brain processes. Otherwise, imparting "slow" brain dynamics to (for instance) changes in connectivity, rather than to (for instance) changes in spike threshold, becomes an arbitrary choice. It is not at all clear, at this stage, what kinds of models can do justice to the complex dynamics of single neurons unfolded in this study. Initial attempts in that direction have been made, linking the complexity of ionic channel proteins to the level of neuronal excitability (Lowen et al., 1999; Marom, 2009; Soudry and Meir, 2010) but are very far from being satisfactory (Marom, 2010).

\section{References}

Abeles M (1991) Corticonics: neural circuits of the cerebral cortex. Cambridge, UK: Cambridge UP.

Adrian ED, Zotterman Y (1926) The impulses produced by sensory nerve endings. 3. Impulses set up by touch and pressure. J Physiol 61:465-483.

Anderson J (1982) Acquisition of cognitive skill. Psychol Rev 89:369-406.

Aston-Jones G, Segal M, Bloom FE (1980) Brain aminergic axons exhibit marked variability in conduction velocity. Brain Res 195:215-222.

Badel L, Lefort S, Brette R, Petersen CC, Gerstner W, Richardson MJ (2008) Dynamic i-v curves are reliable predictors of naturalistic pyramidalneuron voltage traces. J Neurophysiol 99:656-666.

Bakkum DJ, Chao ZC, Potter SM (2008) Long-term activity-dependent plasticity of action potential propagation delay and amplitude in cortical networks. PLoS One 3:e2088.

Ballo AW, Bucher D (2009) Complex intrinsic membrane properties and dopamine shape spiking activity in a motor axon. J Neurosci 29:5062-5074.

Barabási AL (2005) The origin of bursts and heavy tails in human dynamics. Nature 435:207-211.

Bassingthwaighte J, Liebovitch L, West B (1994) Fractal physiology. An American Physiological Society Book. New York: Oxford UP.

Beran J (1994) Statistics for long-memory processes. Boca Raton, FL: Chapman and Hall/CRC.

Brette R, Rudolph M, Carnevale T, Hines M, Beeman D, Bower JM, Diesmann M, Morrison A, Goodman PH, Harris FC Jr, Zirpe M, Natschläger T, Pecevski D, Ermentrout B, Djurfeldt M, Lansner A, Rochel O, Vieville T, Muller E, Davison AP, El Boustani S, Destexhe A (2007) Simulation of networks of spiking neurons: a review of tools and strategies. J Comput Neurosci 23:349-398.

Buzsaki G (2006) Rhythms of the brain. New York: Oxford UP.

Carandini M (2004) Amplification of trial-to-trial response variability by neurons in visual cortex. PLoS Biol 2:E264.

Chen Y, Ding M, Kelso J (1997) Long memory processes ( $1 / f$ type) in human coordination. Phys Rev Lett 79:4501-4504.

Clauset A, Shalizi CR, Newman ME (2009) Power-law distributions in empirical data. SIAM Rev 51:661-703.

De Col R, Messlinger K, Carr RW (2008) Conduction velocity is regulated by sodium channel inactivation in unmyelinated axons innervating the rat cranial meninges. J Physiol 586:1089-1103.

Desai NS, Rutherford LC, Turrigiano GG (1999) Plasticity in the intrinsic excitability of cortical pyramidal neurons. Nat Neurosci 2:515-520.

Drew PJ, Abbott LF (2006) Models and properties of power-law adaptation in neural systems. J Neurophysiol 96:826-833.

Ellerkmann RK, Riazanski V, Elger CE, Urban BW, Beck H (2001) Slow recovery from inactivation regulates the availability of voltage-dependent sodium channels in hippocampal granule cells, hilar neurons and basket cells. J Physiol 532:385-397.

Elul R, Adey W (1966) Instability of firing threshold and "remote" activation in cortical neurones. Nature 212:1424-1425.

Englitz B, Stiefel KM, Sejnowski TJ (2008) Irregular firing of isolated cortical interneurons in vitro driven by intrinsic stochastic mechanisms. Neural Comput 20:44-64.

Esposti F, Ferrario M, Signorini MG (2008) A blind method for the estimation of the hurst exponent in time series: theory and application. Chaos 18:033126.

Esposti F, Signorini MG, Potter SM, Cerutti S (2009) Statistical long-term correlations in dissociated cortical neuron recordings. IEEE Trans Neural Syst Rehabil Eng 17:364-369.

Fairhall AL, Lewen GD, Bialek W, de Ruyter Van Steveninck RR (2001) Efficiency and ambiguity in an adaptive neural code. Nature 412:787-792.

French AS, Torkkeli PH (2008) The power law of sensory adaptation: simulation by a model of excitability in spider mechanoreceptor neurons. Ann Biomed Eng 36:153-161.

Freyer F, Aquino K, Robinson PA, Ritter P, Breakspear M (2009) Bistability and non-Gaussian fluctuations in spontaneous cortical activity. J Neurosci 29:8512-8524.

Friedlander T, Brenner N (2009) Adaptive response by state-dependent inactivation. Proc Natl Acad Sci U S A 106:22558-22563.

Gerstner W, Naud R (2009) How good are neuron models? Science 326: $379-380$.

Gilboa G, Chen R, Brenner N (2005) History-dependent multiple-timescale dynamics in a single-neuron model. J Neurosci 25:6479-6489. 
Gilden DL (2001) Cognitive emissions of 1/f noise. Psychol Rev 108:33-56.

Golomb D, Yue C, Yaari Y (2006) Contribution of persistent $\mathrm{Na}^{+}$current and $\mathrm{M}$-type $\mathrm{K}^{+}$current to somatic bursting in cal pyramidal cells: combined experimental and modeling study. J Neurophysiol 96:1912-1926.

Greenlee MW, Magnussen S (1987) Saturation of the tilt aftereffect. Vis Res 27:1041-1043.

Grossman Y, Parnas I, Spira ME (1979) Mechanisms involved in differential conduction of potentials at high frequency in a branching axon. J Physiol 295:307-322.

Heck D, Rotter S, Aertsen A (1993) Spike generation in cortical neurons: probabilistic threshold function shows intrinsic long-lasting dynamics brain theory: spatio-temporal aspects of brain function. New York: Elsevier Science.

Hodgkin AL, Huxley AF (1952) A quantitative description of membrane current and its application to conduction and excitation in nerve. J Physiol 117:500-544.

Izhikevich EM (2003) Simple model of spiking neurons. IEEE Trans Neural Netw 14:1569-1572.

Izhikevich EM (2004) Which model to use for cortical spiking neurons? IEEE Trans Neural Netw 15:1063-1070.

Jolivet R, Kobayashi R, Rauch A, Naud R, Shinomoto S, Gerstner W (2008) A benchmark test for a quantitative assessment of simple neuron models. J Neurosci Methods 169:417-424.

Kobayashi R, Tsubo Y, Shinomoto S (2009) Made-to-order spiking neuron model equipped with a multi-timescale adaptive threshold. Front Comput Neurosci 3:9.

Liebovitch LS, Fischbarg J, Koniarek JP, Todorova I, Wang M (1987) Fractal model of ion-channel kinetics. Biochim Biophys Acta 896:173-180.

Linkenkaer-Hansen K, Nikouline VV, Palva JM, Ilmoniemi RJ (2001) Long-range temporal correlations and scaling behavior in human brain oscillations. J Neurosci 21:1370-1377.

Linkenkaer-Hansen K, Monto S, Rytsälä H, Suominen K, Isometsä E, Kähkönen S (2005) Breakdown of long-range temporal correlations in theta oscillations in patients with major depressive disorder. J Neurosci 25:10131-10137.

Logan G (1988) Toward an instance theory of automatization. Psychol Rev 95:492-527.

Lowen SB, Teich MC (1992) Auditory-nerve action potentials form a nonrenewal point process over short as well as long time scales. J Acoust Soc Am 92:803-806.

Lowen SB, Teich MC (1996) The periodogram and allan variance reveal fractal exponents greater than unity in auditory-nerve spike trains. J Acoust Soc Am 99:3585-3591.

Lowen SB, Teich MC (2005) Fractal-based point processes. Hoboken, NJ: Wiley-Blackwell.

Lowen SB, Cash SS, Poo M, Teich MC (1997) Quantal neurotransmitter secretion rate exhibits fractal behavior. J Neurosci 17:5666-5677.

Lowen S, Liebovitch L, White J (1999) Fractal ion-channel behavior generates fractal firing patterns in neuronal models. Phys Rev E 59:5970-5980.

Lundstrom BN, Higgs MH, Spain WJ, Fairhall AL (2008) Fractional differentiation by neocortical pyramidal neurons. Nat Neurosci 11:1335-1342.

Magnussen S, Greenlee MW (1985) Marathon adaptation to spatial contrast: saturation in sight. Vis Res 25:1409-1411.

Mainen ZF, Sejnowski TJ (1995) Reliability of spike timing in neocortical neurons. Science 268:1503-1506.

Markram H, Toledo-Rodriguez M, Wang Y, Gupta A, Silberberg G, Wu C (2004) Interneurons of the neocortical inhibitory system. Nat Rev Neurosci 5:793-807.

Marom S (2009) Adaptive transition rates in excitable membranes. Front Comput Neurosci 3:2.

Marom S (2010) Neural timescales or lack thereof. Prog Neurobiol 90:16-28.
Marom S, Shahaf G (2002) Development, learning and memory in large random networks of cortical neurons: lessons beyond anatomy. Q Rev Biophys 35:63-87.

Monto S, Palva S, Voipio J, Palva JM (2008) Very slow EEG fluctuations predict the dynamics of stimulus detection and oscillation amplitudes in humans. J Neurosci 28:8268-8272.

Newell KM, Liu YT, Mayer-Kress G (2001) Time scales in motor learning and development. Psychol Rev 108:57-82.

Nir Y, Fisch L, Mukamel R, Gelbard-Sagiv H, Arieli A, Fried I, Malach R (2007) Coupling between neuronal firing rate, gamma LFP, and BOLD fMRI is related to interneuronal correlations. Curr Biol 17:1275-1285.

Nir Y, Mukamel R, Dinstein I, Privman E, Harel M, Fisch L, Gelbard-Sagiv H, Kipervasser S, Andelman F, Neufeld MY, Kramer U, Arieli A, Fried I, Malach R (2008) Interhemispheric correlations of slow spontaneous neuronal fluctuations revealed in human sensory cortex. Nat Neurosci 11:1100-1108.

Peng CK, Havlin S, Stanley HE, Goldberger AL (1995) Quantification of scaling exponents and crossover phenomena in nonstationary heartbeat time series. Chaos 5:82-87.

Robinson PM (2003) Time series with long memory. New York: Oxford UP.

Rose D, Lowe I (1982) Dynamics of adaptation to contrast. Perception 11:505-528.

Scharf R, Meesmann M, Boese J, Chialvo DR, Kniffki KD (1995) General relation between variance-time curve and power spectral density for point processes exhibiting 1/f beta-fluctuations, with special reference to heart rate variability. Biol Cybern 73:255-263.

Scroggs RS (2008) Evidence of a physiological role for use-dependent inactivation of nav1.8 sodium channels. J Physiol 586:923.

Selz K, Mandell A (1992) Critical coherence and characteristic times in brain stem neuronal discharge patterns. Single neuron computation. San Diego: Academic.

Shahaf G, Eytan D, Gal A, Kermany E, Lyakhov V, Zrenner C, Marom S (2008) Order-based representation in random networks of cortical neurons. PLoS Comput Biol 4:e1000228.

Soen Y, Braun E (2000) Scale-invariant fluctuations at different levels of organization in developing heart cell networks. Phys Rev E Stat Phys Plasmas Fluids Relat Interdiscip Topics 61:R2216-R2219.

Soudry D, Meir R (2010) History dependent dynamics in a generic model of ion channels-an analytic study. Front Comput Neurosci 4:3.

Spira ME, Yarom Y, Parnas I (1976) Modulation of spike frequency by regions of special axonal geometry and by synaptic inputs. J Neurophysiol 39:882-899.

Tal D, Jacobson E, Lyakhov V, Marom S (2001) Frequency tuning of inputoutput relation in a rat cortical neuron in-vitro. Neurosci Lett 300:21-24

Taqqu M, Teverovsky V, Willinger W (1995) Estimators for long range dependence. Fractals 3:785-798.

Teich MC (1989) Fractal character of the auditory neural spike train. IEEE Trans Biomed Eng 36:150-160.

Teich MC, Heneghan C, Lowen SB, Ozaki T, Kaplan E (1997) Fractal character of the neural spike train in the visual system of the cat. J Opt Soc Am A Opt Image Sci Vis 14:529-546.

Toib A, Lyakhov V, Marom S (1998) Interaction between duration of activity and time course of recovery from slow inactivation in mammalian brain $\mathrm{Na}^{+}$channels. J Neurosci 18:1893-1903.

Turrigiano G, Abbott LF, Marder E (1994) Activity-dependent changes in the intrinsic properties of cultured neurons. Science 264:974-977.

van Pelt J, Wolters PS, Corner MA, Rutten WL, Ramakers GJ (2004) Longterm characterization of firing dynamics of spontaneous bursts in cultured neural networks. IEEE Trans Biomed Eng 51:2051-2062.

Wink AM, Bullmore E, Barnes A, Bernard F, Suckling J (2008) Monofractal and multifractal dynamics of low frequency endogenous brain oscillations in functional mri. Hum Brain Mapp 29:791-801. 